\title{
Density of rational points on elliptic fibrations-II
}

by

\author{
Ritabrata Munshi (Piscataway, NJ)
}

1. Introduction. A rational elliptic surface over $\mathbb{P}^{1}(\mathbb{Q})$ has a Weierstrass model given by

$$
Y^{2}=X^{3}+g_{4}(S, T) X+g_{6}(S, T),
$$

where $g_{4}$ is a homogeneous polynomial of degree 4 and $g_{6}$ is a homogeneous polynomial of degree 6 over the field of rationals (see [5]). The equation (1) defines a sextic hypersurface $V$ in the weighted projective space $\mathbb{P}(1,1,2,3)$. With any rational point $P$ on $V$ with reduced coordinates $(S, T, X, Y)$ we associate the height

$$
H(P)=\max \left\{|S|,|T|,|X|^{1 / 2},|Y|^{1 / 3}\right\} .
$$

With respect to this height we define the counting function

$$
N(H)=\#\{P \in V(\mathbb{Q}): H(P) \leq H\} .
$$

We wish to understand the behaviour of $N(H)$ as $H$ tends to infinity. The surface $V$ may contain exceptional divisors defined over $\mathbb{Q}$, and any such divisor has $\asymp H^{2}$ rational points of height $H$. (For example, if $g_{6}=0$ then $X=Y=0$ is an exceptional divisor.) It appears that these contributions (when present) play the dominant role in determining the behaviour of $N(H)$. To be more precise, let

$$
U=V-\{\text { exceptional divisors }\}
$$

be the open complement of the set of exceptional divisors, and define the refined counting function

$$
N(H, U)=\#\{P \in U(\mathbb{Q}): H(P) \leq H\} .
$$

We may regard $N(H, U)$ as counting "non-trivial" points on $V$. Manin et al. (see [1], [2]) have given some precise conjectures concerning the asymptotic behaviour of $N(H, U)$, but in this paper we are concerned with the following weaker version of the conjecture.

2000 Mathematics Subject Classification: 11D45, 14G05.

Key words and phrases: elliptic pencil, rational points. 
Conjecture 1. For any $\varepsilon>0$, we have

$$
N(H, U) \ll H^{1+\varepsilon},
$$

where the implied constant depends on the variety $V$ and $\varepsilon$.

In our previous paper [6] we established some rough estimates towards this conjecture for certain special elliptic fibrations. In particular, for the surface given by

$$
Y^{2}=X^{3}+Q(S, T)^{3},
$$

where $Q(S, T)$ is a positive definite quadratic form defined over $\mathbb{Z}$, we proved that

$$
N(H, U) \ll H^{4 / 3+\varepsilon} .
$$

This was obtained by counting the number of integer solutions of $Y^{2}=$ $X^{3}+Z^{3}$, via reduction to a pair of quadratic equations and an application of a uniform bound of Heath-Brown on the density of rational points on plane conics.

On the other hand, from our recent work with Iwaniec [4], one may establish the almost sharp lower bound

$$
N(H, U) \gg \frac{H}{(\log H)^{3 / 2}}
$$

for the surface

$$
Y^{2}=X^{3}+a Q(S, T)^{2} X+b Q(S, T)^{3},
$$

where $Q$ is a quadratic form. (In [4] we assume that the cubic $X^{3}+a X+b$ is irreducible.) So it is desirable to obtain good upper bounds for $N(H, U)$ in the case of the surface given by (5).

We note that from the geometric point of view, the surfaces given by (5) are precisely the rational elliptic surfaces with two conjugate double points (see [5]). In this paper we deal with such surfaces. We also assume that the associated cubic form

$$
f(X, Z)=X^{3}+a Z^{2} X+b Z^{3}
$$

factorizes as a product of a linear form and a positive definite quadratic form. Hence after a change of variable we may assume

$$
f(X, Z)=(X+\lambda Z)(X+\delta Z)(X+\bar{\delta} Z),
$$

where $\lambda \in \mathbb{Z}$, and $\delta$ is a generator of the ring of integers of an imaginary quadratic field. As in [6], the problem of estimating $N(H, U)$ boils down to counting the number of integer solutions of the equation $Y^{2}=f(X, Z)$ with the restriction $|X|<H^{2}$ and $|Z|<H^{2}$. Now we state our main theorem. 
TheOrem 2. Suppose the cubic $f(X, Z)$ satisfies (6). Then the number of integer solutions to the equation $Y^{2}=f(X, Z)$ with the restrictions

$$
0<|X|,|Z|<H^{2} \quad \text { and } \quad Y \neq 0
$$

is bounded by $O_{f, \varepsilon}\left(H^{5 / 4+\varepsilon}\right)$.

From the above theorem we conclude the following bound for the density of rational points on the surface (5).

Corollary 3. Suppose the cubic $f(X, Z)$ satisfies (6), and $Q$ is a positive definite binary quadratic form defined over $\mathbb{Z}$. Let $V$ be the surface

$$
Y^{2}=f(X, Q(S, T)) .
$$

Then $N(H, U)=O_{f, Q, \varepsilon}\left(H^{5 / 4+\varepsilon}\right)$.

Proof. The corollary follows by setting $Z=Q(S, T)$, and observing that there are at most $O\left(H^{\varepsilon}\right)$ pairs $(S, T)$ for each fixed $Z$.

REMARK 1. We observe that the cubic associated to the surface (3) satisfies (6). Hence the above corollary improves our earlier result (4).

2. A lemma. For simplicity we assume that in (6) we have $\delta=\sqrt{D}$ where $D$ is a square-free negative number congruent to 2 , or 3 modulo 4 . Let $K=\mathbb{Q}(\sqrt{D})$ be the imaginary quadratic field and $O_{K}=\mathbb{Z}[\sqrt{D}]$ the ring of integers. Then the norm form is given by $\mathbf{N}(a+\sqrt{D} b)=a^{2}-D b^{2}$. Suppose $h$ is the class number and let $\mathcal{R}$ be a set of integral representatives of the classes such that the norm of each ideal in $\mathcal{R}$ satisfies the Minkowski bound.

Let $d_{1}$ and $d_{2}$ be a pair of coprime square-free positive rational integers, and let $d=d_{1} d_{2}$. Consider the following pair of equations:

$$
\begin{aligned}
& d_{1} y_{1}^{2}=x+\lambda z, \\
& d_{2} y_{2}^{2}=x^{2}-D z^{2},
\end{aligned}
$$

in four variables $x, z, y_{1}$ and $y_{2}$. Our goal in this section is to estimate the number $N\left(H ; d_{1}, d_{2}\right)$ of integer solutions of the above pair of equations with the restrictions $0<|x|,|z|<H, y_{i} \neq 0$, and $(x, z)=1$.

LEMma 4. We have

$$
N\left(H ; d_{1}, d_{2}\right) \ll_{\lambda, D, \varepsilon}\{1+\sqrt{H / d}\}(H d)^{\varepsilon}
$$

for any $\varepsilon>0$.

Proof. First, we obtain a set of parametric solutions of (8). Substituting these in (7) we get a set of conics. Then we apply a result of Heath-Brown (Lemma 5 below) to count the number of points on these conics.

Let $\left(y_{2}, x, z\right)$ be a solution of (8). Since $x$ and $z$ are coprime, it follows that $(x+\sqrt{D} z)$ and $(x-\sqrt{D} z)$ are coprime except for a possible common 
factor $l \mid 4 D$. Hence from equation (8) we conclude that

$$
(x+\sqrt{D} z) O_{K}=\mathbf{u v}^{2},
$$

where $\mathbf{u}$ is square-free and each prime ideal appearing on the right hand side is either split or ramified. Consequently,

$$
\mathbf{N u}=d_{2} \text { and } \mathbf{N v}=y_{2} .
$$

Now we have ideals $I$ and $J$ in the set $\mathcal{R}$ of integral representatives of ideal classes, such that

$$
I \mathbf{u}=u O_{K} \quad \text { and } \quad J \mathbf{v}=v O_{K}
$$

for some algebraic integers $u=a+\sqrt{D} b$ and $v=M+\sqrt{D} N$, respectively. From (9) and (11) we see that $I J^{2}=(e+\sqrt{D} f) O_{K}$ is principal. Then it follows that

$$
(x+\sqrt{D} z)(e+\sqrt{D} f)=\alpha(a+\sqrt{D} b)(M+\sqrt{D} N)^{2}
$$

for some unit $\alpha$ in $O_{K}$. Without loss of generality, we may assume that $\alpha=1$. Then we get

$$
\begin{gathered}
e x+D f z=a\left(M^{2}+D N^{2}\right)+2 b D M N \\
f x+e z=2 a M N+b\left(M^{2}+D N^{2}\right) .
\end{gathered}
$$

Solving this system of linear equations we can express $x, z$ as quadratics in $M, N$ with coefficients depending on $a, b, e, f$ and $D$. We note that there are at most $O_{D, \varepsilon}\left(d^{\varepsilon}\right)$ choices for $(a, b)$, and at most $O_{D}(1)$ choices for $(e, f)$. So we conclude that if $\left(y_{2}, x, z\right)$ is a solution of $(8)$, then $(x, z)$ satisfies the equations (12), (13) for some choice of $(a, b, e, f)$.

Now, substituting the values of $x$ and $z$ in the equation (7), we get

$$
E d_{1} y_{1}^{2}=A M^{2}+2 B M N+C N^{2},
$$

where $E=e^{2}-D f^{2}$, and the other coefficients are as follows:

$$
\begin{aligned}
& A=(e a-D f b)+\lambda(e b-f a), \\
& B=D(e b-f a)+\lambda(e a-D f b), \\
& C=D(e a-D f b)+\lambda D(e b-f a) .
\end{aligned}
$$

It remains to count the number of solutions of (14). First, it is crucial to note that there are restrictions on the possible values of $\operatorname{gcd}\left(M, N, y_{1}\right)$. Using the coprimality of $x$ and $z$, and equations (12), (13), we observe that $\operatorname{gcd}(M, N)$ divides $E$. So we are only concerned with those solutions of (14) such that $\operatorname{gcd}\left(M, N, y_{1}\right) \mid E$. The set of all such integers $E$, and hence the set of all possible values for $\operatorname{gcd}\left(M, N, y_{1}\right)$, is determined by the fixed set of representatives $\mathcal{R}$. This means that the number of solutions of (14) that contribute to $N\left(H ; d_{1}, d_{2}\right)$ is, up to a power of $D$, the same as the number 
of primitive solutions (i.e. $\operatorname{gcd}\left(M, N, y_{1}\right)=1$ ) of (14) in the box

$$
\left|y_{1}\right| \ll_{\lambda} \sqrt{H} / \sqrt{d_{1}}, \quad|M|,|N| \ll_{D} \sqrt{H} / d_{2}^{1 / 4} .
$$

These inequalities follow from the equations (7) and (9). To count the primitive solutions we use the following result of Heath-Brown [3].

Lemma 5 (Heath-Brown). Let $q$ be a ternary quadratic form with matrix $\mathcal{M}$. Let $\Delta=|\operatorname{det} \mathcal{M}|$, and assume that $\Delta \neq 0$. Write $\Delta_{0}$ for the highest common factor of the $2 \times 2$ minors of $\mathcal{M}$. Then the number of primitive integer solutions of $q(x)=0$ in the box $\left|x_{i}\right|<R_{i}$ is

$$
\ll_{\varepsilon}\left\{1+\left(R_{1} R_{2} R_{3} \Delta_{0}^{2} / \Delta\right)^{1 / 3+\varepsilon}\right\}\left(R_{1} R_{2} R_{3}\right)^{\varepsilon}
$$

for any $\varepsilon>0$.

We apply the above result to the equation (14). In this case the absolute value of the determinant is given by

$$
d_{1}\left|E\left(B^{2}-A C\right)\right|=d_{1}\left(\lambda^{2}-D\right) \mathbf{N} u\left(\mathbf{N} I J^{2}\right)^{2} \geq d .
$$

Also the gcd of all $2 \times 2$ minors is $E$, which is $O_{D}(1)$. Hence, in the notations of Lemma 5 , we have $\Delta \geq d$ and $\Delta_{0}=O_{D}(1)$. Also from (15) we get

$$
R_{1} R_{2} R_{3} \ll_{\lambda, D} H^{3 / 2} / d^{1 / 2} .
$$

So it follows from Lemma 5 that the number of solutions of (14) contributing to $N\left(H ; d_{1}, d_{2}\right)$ is

$$
\ll_{\lambda, D, \varepsilon}\{1+\sqrt{H / d}\}(H d)^{\varepsilon} .
$$

REMARK 2. We note that the exponent of $H$ in Lemma 4 is sharp. In fact, it follows that

$$
N\left(H ; d_{1}, d_{2}\right) \gg_{\lambda, D, d} \sqrt{H}
$$

if any of the conics (14) has a rational point. For example, consider the system (7), (8) with $d_{1}=d_{2}=\lambda=-D=1$. Then a set of solutions of (8) is given by taking $\left(x, z, y_{2}\right)=\left(a^{2}-b^{2}, 2 a b, a^{2}+b^{2}\right)$. Substituting this in (7), we get a conic

$$
y_{1}^{2}=a^{2}-b^{2}+2 a b .
$$

Clearly $\left(y_{1}, a, b\right)=(1,1,0)$ is a point on the conic, and then we can construct $\gg \sqrt{H}$ rational points which contribute to $N\left(H ; d_{1}, d_{2}\right)$. The author wishes to thank the referee for this observation.

3. Proof of Theorem 2. Suppose $(X, Y, Z)$ is an integer triplet satisfying the equation

$$
Y^{2}=f(X, Z),
$$

and such that $Y \neq 0$ and $0<|X|,|Z|<H^{2}$. We write $g=\operatorname{gcd}(X, Z)$ in the form $g=d t^{2}$, where $d$ is square-free. It follows from (16) that $d^{2} t^{3} \mid Y$. 
Defining

$$
x=\frac{X}{g}, \quad z=\frac{Z}{g}, \quad y=\frac{Y}{d^{2} t^{3}},
$$

we observe that the integer triplet $(x, y, z)$ satisfies the equation

$$
d y^{2}=f(x, z) \quad \text { with } \quad(x, z)=1 .
$$

Also, we have $d<(H / t)^{3 / 2}$ and the size restrictions

$$
0<|x|,|z|<\frac{1}{d}\left(\frac{H}{t}\right)^{2} \text { and } 0<|y| \ll_{f} \frac{1}{d^{2}}\left(\frac{H}{t}\right)^{3} .
$$

Our aim is to count the number of integer solutions of the equation (17) for any given square-free $d$, satisfying the above restriction on the sizes. As the form of the equation does not depend on the positive integer $t$, we do the counting by writing $H$ in place of $H / t$, and then at the end we replace $t$ back and sum over the range $0<t<H$. However from the form of the estimate it will be clear that this sum over $t$ only increases the bound by a constant and does not alter the magnitude.

Using the assumed structure (6) of the cubic form $f$, we observe that $d$ and $y$ factorize as $d=d_{1} d_{2}$ and $y=y_{1} y_{2}$ so that

$$
\begin{aligned}
& d_{1} y_{1}^{2}=x+\lambda z, \\
& d_{2} y_{2}^{2}=\mathbf{N}(x+\sqrt{D} z) .
\end{aligned}
$$

Now we apply the bound from Lemma 4 to count the number of solutions when $d$ is in the range $d<H^{5 / 4}$. It follows that

$$
\sum_{\substack{0<|X|,|Z|<H^{2} \\ 0<Y^{2}=f(X, Z) \\ d<H^{5 / 4}}} 1 \ll_{f, \varepsilon} \sum_{d<H^{5 / 4}}\left\{1+\frac{H}{d}\right\}(H d)^{\varepsilon} \ll_{f, \varepsilon} H^{5 / 4+\varepsilon} .
$$

For larger values of $d$ we count by switching the "parameters" and the "variables". To this end we combine the equations (18), (19) and obtain

$$
d_{2} y_{2}^{2}=\left(y_{1}^{4}\right) d_{1}^{2}-\left(2 \lambda y_{1}^{2}\right) d_{1} z+\left(\lambda^{2}-D\right) z^{2} .
$$

We consider this as an equation in $y_{2}, d_{1}$ and $z$, with parameters $d_{2}$ and $y_{1}$. Now given $d_{2}$ and $y_{1}$, we restrict $d_{1}$ to the dyadic segment $[L, 2 L]$. Then we note that we have the following bounds:

$$
0<\left|y_{2}\right| \ll_{f} \frac{H^{2}}{L d_{2}^{3 / 2}} \quad \text { and } \quad 0<|z| \ll \frac{H^{2}}{L d_{2}} .
$$

Observe that the bound on $y_{2}$ follows from (19). Also $\operatorname{gcd}\left(y_{2}, d_{1}, z\right)=1$, as otherwise equation (18) will imply that $\operatorname{gcd}(x, z)>1$. In the context of 
equation (21), using the notations of Lemma 5, we note that

$$
R_{1} R_{2} R_{3} \ll_{f} \frac{H^{4}}{L d_{2}^{5 / 2}}, \quad \Delta \gg_{f} d_{2} y_{1}^{4}, \quad \Delta_{0} \ll_{f} 1 .
$$

So by Lemma 5 , the number of solutions of (21) is

$$
\ll_{f, \varepsilon}\left\{1+\frac{H^{4 / 3}}{L^{1 / 3} d_{2}^{1 / 6} d_{2} y_{1}^{4 / 3}}\right\} H^{\varepsilon} .
$$

Now since $d_{1} d_{2}>H^{5 / 4}$, we get

$$
L^{1 / 3} d_{2}^{1 / 6} \gg H^{5 / 12} d_{2}^{-1 / 6}
$$

Combining this with the inequality $d_{2} \ll_{f} H^{4 / 3}$ (which follows from (19)), we find that the expression in $(22)$ is

$$
\ll_{f, \varepsilon}\left\{1+\frac{H^{41 / 36}}{d_{2} y_{1}^{4 / 3}}\right\} H^{\varepsilon} .
$$

Then adding the contribution of all the dyadic blocks, we conclude that the number of solutions with $d_{2} y_{1} \leq H^{5 / 4}$ is at most $O_{f, \varepsilon}\left(H^{5 / 4+\varepsilon}\right)$.

Now it remains to count the number of solutions of (16) with

$$
d_{1} d_{2}>H^{5 / 4} \quad \text { and } \quad d_{2} y_{1}>H^{5 / 4} \text {. }
$$

Multiplying the above inequalities we get $d_{1} y_{1} d_{2}^{2}>H^{5 / 2}$. From the size restriction and equation (18) we get $d_{1} y_{1}^{2} \ll_{f} H^{2} / d$, and hence $d_{1} y_{1} \ll_{f}$ $H / \sqrt{d_{2}}$. It follows that

$$
\frac{H d_{2}^{2}}{\sqrt{d_{2}}} \gg_{f} d_{1} y_{1} d_{2}^{2}>H^{5 / 2}
$$

i.e. $d_{2} \gg_{f} H$. The number of possible values for $z$ is at most $H^{2} / d \ll_{f} H^{3 / 4}$. Also from the bound $d_{1} y_{1} \ll_{f} H / \sqrt{d_{2}} \ll_{f} \sqrt{H}$, it follows that the number of possible pairs $\left(d_{1}, y_{1}\right)$ is

$$
\ll_{f} \sum_{n \leq \sqrt{H}} \sum_{d_{1} y_{1}=n} 1 \ll_{f, \varepsilon} H^{1 / 2+\varepsilon} .
$$

Hence under (23) the number of possible values that the triplet $\left(d_{1}, y_{1}, z\right)$ may take is at most $O_{f, \varepsilon}\left(H^{5 / 4+\varepsilon}\right)$. Also we notice that $x$ is uniquely determined from the triplet using equation (18), and $d_{2}, y_{2}$ are uniquely determined (as $d_{2}$ is square-free) from equation (19). Hence the number of solutions under the restriction $(23)$ is bounded by $O_{f, \varepsilon}\left(H^{5 / 4+\varepsilon}\right)$. The theorem follows. 


\section{References}

[1] V. V. Batyrev et Yu. I. Manin, Sur le nombre des points rationnels de hauteur borné des variétés algébriques, Math. Ann. 286 (1990), 27-43.

[2] J. Franke, Yu. I. Manin and Y. Tschinkel, Rational points of bounded height on Fano varieties, Invent. Math. 95 (1989), 421-435.

[3] D. R. Heath-Brown, The density of rational points on curves and surfaces, Ann. of Math. (2) 155 (2002), 553-595.

[4] H. Iwaniec and R. Munshi, Cubic polynomials and quadratic forms, preprint, 2007.

[5] R. Miranda, The moduli of Weierstrass fibrations over $P^{1}$, Math. Ann. 255 (1981), 379-394.

[6] R. Munshi, Density of rational points on elliptic fibrations, Acta Arith. 129 (2007), $63-70$.

Department of Mathematics

Rutgers University

Hill Center, Busch-Campus

110 Frelinghuysen Road

Piscataway, NJ 08854, U.S.A.

E-mail: rmunshi@math.rutgers.edu

Received on 2.11.2007

and in revised form on 30.1.2008 